\title{
Control Modeling of a New Type Electric Mechanical Continuously Variable Transmission and Realization of the Control Objectives
}

\author{
Xiumin Yang, ${ }^{1, *}$, Song Zhang ${ }^{1}$, Zeqi Wang ${ }^{2}$ and Lei Zhang ${ }^{2}$ \\ ${ }^{1}$ Department of Automatic Controls, Shenyang Institute of Engineering, Shenyang 110136, China \\ ${ }^{2}$ Mechanical Engineering and Automation School, Northeastern University, Shenyang 110004, P. R. China \\ *Corresponding author
}

\begin{abstract}
A new electric mechanical metal belt (chain) continuously variable transmission (EMCVT) for vehicles was studied in this paper. With the use of direct-current (DC) motor controlling the rolling screw, the displacement of the taper disc pressed by the Belleville spring can be controlled, which achieves the function of speed governing. To make sure that the control objectives are realized, the taper disc clamp force control model of the Belleville spring was established by analyzing the speed ratio control model and studying the axial thrust ratio between the driving taper disc and the driven taper disc. So the association model between the CVT transmission ratio under the steady-state condition and the engine speed was proposed. The economic and driving force characteristic curve were determined by tests, and the EMCVT was controlled with the method of the incompletion differential PID control. The validity of models and control strategies were proved by bench tests and road tests.
\end{abstract}

Keywords-electric -mechanical continuously variable transmission (EMCVT); control model; axial thrust ratio; clamp force control; speed ratio control

\section{INTRODUCTION}

The current vehicle metal belt (chain) continuously variable transmissions generally adopt the hydraulic pressure device or electric hydraulic pressure system as the control plan. However, using the pump, the valve and the hydraulic torque converter to control the clamp force and the transmission ratio makes the efficiency reduced and failure rate improved. Meanwhile, the vehicle actual fuel consumption is still very high. In this paper, the Electric-Mechanical Continuously Variable Transmission (EMCVT) works through the metal belt (chain), abandoning the low efficient pumps, valves and other hydraulic elements. Instead, motors, gears and Belleville springs are applied to the EMCVT, which not only reduces the energy consumption and the cost, but also improve the reliability.

As a result of using the speed governing method of the rolling screw drive, the Belleville spring prepressing clamp and the taper disc displacement control, the speed ratio relation and the clamp force characteristic directly influence the control strategies and the control efficiency in this new EMCVT.

\section{MECHANICAL SySTEM STRUCTURE OF EMCVT}

The new EMCVT is composed of the front case, the middle case, the rear case, the clutch control motor and the speed ratio control motor. The three cases mentioned above are all made of cast aluminum, ensuring the entire system much lighter in the premise of meeting the strength requirements. The clutch control motor consists of the permanent magnet DC motor, the self-locking worm gear reducer, the spring compensation mechanism, the position sensor, the mechanical position limitation mechanism, inhaul cables and so on. When the clutch motor is rotating forward or reversely, the straight reciprocating motion can be achieved by using the worm gear mechanism and lever mechanism. The power is transmitted by the inhaul cables.

The structure of the speed ratio control motor is shown in Figure 1. The permanent magnet DC motor that the rated voltage is $12 \mathrm{~V}$ with the contracting brake device is chosen as the speed ratio control motor. Through the synchromesh toothed belt and the dual-range reduction gear, the clamp force used to control the speed ratio is applied on the driving taper disc by the ball screws and the Belleville springs. Then the pressure between the belts and the driven taper disc Belleville springs reaches a new state of equilibrium. Thus, the speed ratio of the transmission is controlled. The driving wheel and the driven wheel both consist of the fixed taper disc and the movable taper disc. The power between the fixed and movable taper discs is transmitted through the metal belt. During the operation of the mechanism, the movable taper discs of driving wheels and driven wheels produce the axial displacements under the Belleville springs force.

From the transmission principle of the EMCVT, we can know that EMCVT can continuously and arbitrarily change the transmission ratio in the entire design transmission ratio area. At the same time, with the motor and the deceleration system replaced by the traditional CVT that adopts the hydraulic system, the overall efficiency of the EMCVT has a significant improvement. Currently, this transmission has completed the bench tests and passed the tests from National Motor Vehicle Quality Supervision and Inspection Center (Chongqing, China). (Report NO. 10-WT-DBJ-N76). Meanwhile, it has also passed the technical appraisement from China Machinery Industry 
Federation. Now, the transmission has installed on the vehicles, and carried out a series of other tests.

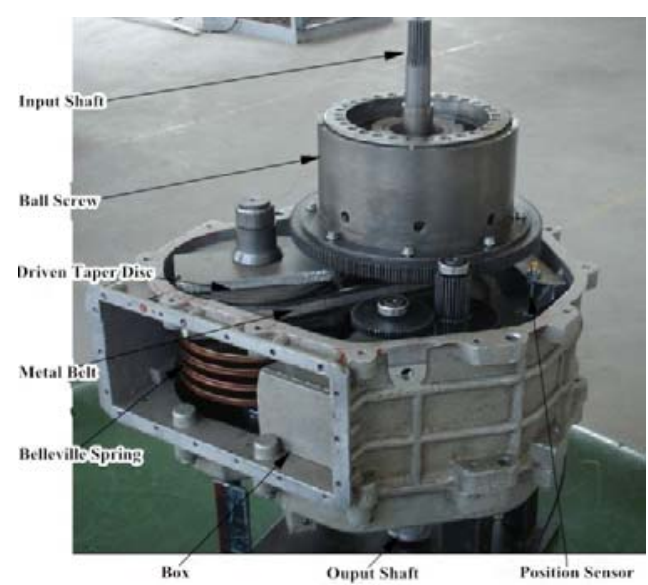

(a) The mechanism structure

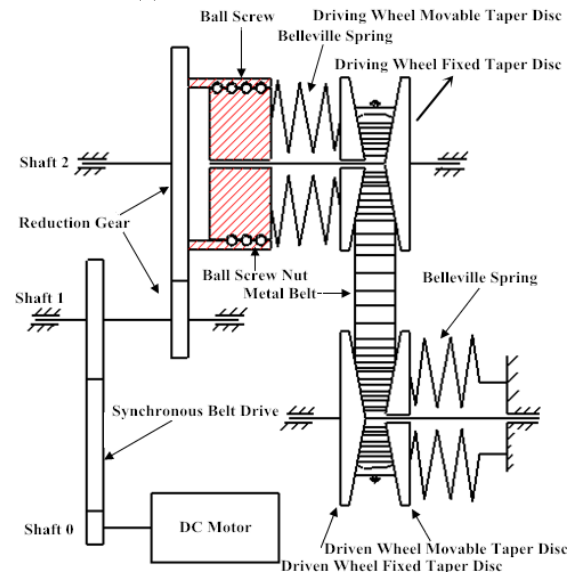

(b) The transmission principle of the EMCVT

FIGURE I. THE MECHANISM STRUCTURE AND THE TRANSMISSION PRINCIPLE OF THE EMCVT

\section{Modeling of EmcVt Speed Ratio Control System}

As is shown in Fig.1, the transmission ratio of this EMCVT is changed by changing the radius of the metal belt powered by the taper disc. In turns, the movement of the taper disc is achieved by the movement of the nuts, and the nuts are driven by the screw rotation. The screw rotation is driven by the tri-range cylindrical gear reduction mechanism powered by the DC motor. Obviously, it's the DC motor that achieves the goal of speed governing.

\section{Speed Ratio Model of the CVT}

From the mechanical structure of the EMCVT, the transmission ratio can be determined by the driving wheel pitch radius $\left(R_{D R}\right)$ and the driven wheel pitch radius $\left(R_{D N}\right)$,

During the process of the transmission, because the length ( $L$ ) of the metal belt can be regarded as a constant (the variation caused by the elastic deformation is very small), so we can know that,

$$
L=(\pi+2 \alpha) R_{D R}+(\pi-2 \alpha) R_{D R}+2 d \cos \alpha
$$

Where $\alpha \approx \sin \alpha=\left(R_{D R}-R_{D N}\right) / d, d$ is the center distance of the transmission shaft. From the equation (1), the approximate calculation equation of the speed ratio can be determined $^{[1]}$,

$$
i=\frac{-B \pm \sqrt{B^{2}-4 A C}}{2 A}
$$

In equation (2), $A=R_{D R} / d, B=\pi-2 R_{D R} / d$, $C=\pi+R_{D R} / d+2 d / R_{D R}-L / R_{D R}$. The speed ratio of the metal belt continuously variable transmission device ranges from 0.5 to 2.5 .

\section{Axial Thrust Ratio $\varphi_{\text {Between Driving Taper DisC }}$ AND DRIVEN TAPER DISC}

The axial thrust ratio $\varphi$ is an important parameter in terms of the EMCVT design calculation and the entire control system, that is $\varphi=F_{a A} / F_{a B}$. The experiments show that the axial thrust ratio $\varphi$ changes with the transmission ratio $i$ and the torque ratio $\lambda=T_{e} / T_{e \max }$. After further experimental analysis, we can know that the axial thrust ratio $\varphi$ is mainly determined by the transmission ratio $i$ and the torque ratio $\lambda$. The maximum torque that the mechanism can transfer has a very little impact on the axial thrust ratio $\varphi$, especially under the condition that the torque ratio $\lambda>0.5, \lambda$ almost has no impact on the $\varphi$. However, when the CVT works normally, the actual torque ratio generally is higher than 0.5. Thus, the influence from the torque ratio $\lambda$ can be ignored.

The relations among the axial thrust are calculated under the circumstance of not transferring torque. We use $F_{\text {asma11 }}$ to represent the axial thrust when the belt wheel meshed with the belt in the small working radius $R_{\text {small }}$. Thus, the radical force per unit arc length of the metal steel ring in the V-gutter is represented by $P_{T}$ and the relation between the initial tension of the steel ring and the axial thrust of the belt wheel is

$$
4 T_{0} \cos \lambda=2 \int_{0}^{\frac{\pi}{2}-\lambda} P_{T} \times \cos \theta \times\left(R_{\mathrm{smal1}}+\Delta h\right) d \theta
$$




$$
=\frac{4 \times F_{a \text { sma11 }} \times \tan \alpha}{(\pi-2 \lambda)} \times \cos \lambda
$$

Where, $\alpha$ is the cone angle of the taper disc. $\Delta h$ is the distance between the pendulum edge of the thrust sheet and the saddle surface. The relation between the belt wheel axial thrust is

$$
\varphi=\frac{F_{a \text { smal1 }}}{F_{a \text { big }}}=\frac{(\pi-2 \lambda) \times \tan \alpha^{\prime}}{(\pi+2 \lambda) \times \tan \alpha}
$$

In terms of the actual control of the CVT, the most intuitive and effective method is to calibrate the relations among axial thrusts of driving wheels and driven wheels. Then, the experimental results are collected to make the data map.

\section{Axial Clamp Force Model of CVt Driving AND DRIVEN WHEELS}

In the process of speed governing, the clamp force $F_{\text {diskR }}$, $F_{\text {diskN }}$ of the driving and driven wheel both has the function relationship with the taper discs deformation. With an arbitrary ratio $i$, the distance between the driving shaft movable taper disc and its initial position is $S_{1}$, similarly, the distance between the driving shaft movable taper disc and its initial position is $S_{2}$. So we can acquire the value of the spring force of the driving and driven taper discs during the process of the speed governing.

$$
\left\{\begin{array}{l}
F_{d i s k R}=F_{d i s k R}\left(k-\frac{s_{1}}{8}\right) \\
F_{d i s k N}=F_{d i s k N}\left(k^{\prime}+\frac{s_{2}}{8}\right)
\end{array}\right.
$$

Where,

$$
\begin{gathered}
s_{1}=2\left(R_{D R}-R_{D R \min }\right) \tan \alpha, \\
s_{2}=2\left(R_{D N \text { max }}-R_{D N}\right) \tan \alpha .
\end{gathered}
$$

According to the relations among the driving taper disc, the driven taper disc and the transmission ratio, we can acquire that

$$
S_{2}=2 \tan \alpha\left[R_{2 \max }-i\left(\frac{S_{1}}{2 \tan \alpha}+R_{1 \text { min }}\right)\right]
$$

\section{ASSOCIATION MODEL OF THE CVT TRANSMISSION RATIO AND ENGINE SPEED UNDER STEADY-STATE CONDITIONS}

The definition of the steady-state conditions: all parameters of the vehicle power transmission system are all constant, such as the throttle opening, the speed, the torque, the wheel speed, the frontal drag, the road gradient and so on. The control of the CVT is relatively easy under the steady-state conditions.

Assuming that the clutch is in a state of the lock-up, that is, there is no belt slip when the vehicle is running at the drive shift. Thus, the angular velocity of the engine crankshaft $\omega_{e}$ is equal to the angular velocity of the driving wheel $\omega_{p}$. The ratio of the CVT is

$$
i=\frac{\omega_{p}}{\omega_{s}}=\frac{T_{s}}{T_{p} \eta}
$$

In the above equation, $T_{p}$ represents the torque applied to the driving wheel from the metal belt. $T_{s}$ represents the torque applied to the driven wheel from the metal belt. $\eta$ is the transmission ratio of the CVT. Under the condition that the vehicle is running at a steady speed, we can acquire the power of the vehicle from the engine characteristics curve.

$$
P=\omega_{e} \cdot T_{e}=\omega_{d} \cdot T_{d}
$$

Where, $P$ is the power of the vehicle. $T_{e}$ is the engine output torque. $T_{d}$ is the equivalent drag torque of the driving shaft.

Because we know that $T_{P}=T_{e}, T_{S}=T_{e} \eta_{a}, \omega_{d}=i_{a} \omega_{S}$, $\omega_{e}=\omega_{P}$, we can acquire the relation equation of the CVT transmission ratio and the engine speed in the steady-state working condition.

$$
i \omega_{e}=\frac{P \eta_{a}}{T_{p} \eta}
$$

In equation (9), $i_{a}$ is the transmission ratio from the driven wheel to the driving shaft. $\eta_{a}$ is the effectiveness from the driven wheel to the driving shaft. In the steady-state conditions, the engine optimizing dynamic performance and economic performance shift curve can be converted into the engine optimizing dynamic speed ratio performance and economic performance curve. That lays the foundation of controlling the speed ratio. 


\section{CONTROL STRATEGIES OF EMCVT}

The economic gear shift is taken as the example to illustrate the control strategy. When the vehicle is driving under the economic gear shift, the signals of the current throttle and the engine speed will be read. Whether the current engine speed is equal to the optimizing economic speed corresponding to the current throttle is the basis for determining the gear shift is chosen as the economic gear shift. If the engine speed meets the above judgement, a new throttle signal will be detected to repeat the above process. If not, the signal will go back to the main procedure. According to the equation (9), we know that the engine speed is in inverse proportion of the transmission ratio. If the current engine speed is lower than the current throttle optimizing economic speed, we have to judge that the transmission ratio is whether in a state of the maximum position. At that time, the transmission ratio is supposed to reduce to increase the engine speed. If the transmission is in a state of the minimum position, the transmission ratio is supposed to remain unchanged. If the current engine speed is higher than the current throttle optimizing economic speed, we have to judge that the transmission ratio is whether in a state of the minimum position. At that time, we have to judge that the transmission ratio is whether in a state of the maximum position. If the transmission is in a state of the maximum position, the transmission ratio is supposed to remain unchanged. After detecting the transmission ratio, the engine speed will be detected in a new round. Similarly, the dynamic gear shift has almost the same control strategy. The only difference is that the engine speed has to compare with the optimizing dynamic speed.

During the process of the vehicle driving, all kinds of disturbances occur successively. Thereby, the traditional incremental PID is not suitable to choose. In order to solve this problem, the incomplete differential PID control is adopted, adding a first-order inertia process (the low-pass filter), $G_{f}(s)=1 /\left[1+T_{f}(s)\right]$. Currently, there are two kinds of incomplete differential PID control methods. One is adding the first-order inertia process to the differential link, and the other one is adding the first-order inertia process to the PID controlling unit. Because of relying on the same principle, we will illustrate the first control method. For the first structure, the transfer function is

$$
u_{D}(k)=\frac{T_{f}}{T_{f}+T} u_{D}(k-1)+\frac{K_{P} T_{D}}{T_{f}+T}[e(k)-e(k-1)]
$$

From above equations, we can find that the height of the control pulsing signals from the differential output in the first sampling period all reduce in certain degree after introducing the incomplete differential. In the following periods, the height will gradually reduce according to the law of $a^{k} u_{D}(0)$. Thus, the problem of lacking in incremental PID control is solved, and now it has excellent control characteristics.

\section{CONCLUSION}

EMCVT is a new vehicle continuously variable transmission. By adopting the DC motor to control the ball screws, the displacement of the taper disc compressed by the Belleville springs. Thus, the function of speed governing is realized. For this special structure, the speed ratio control model, the clamp force control model and the speed governing under the steady-state working conditions are all established. Meanwhile, all of the models are all verified by the comparison bench tests in the later stage. The control strategies of the EMCVT are also verified by bench tests and road tests. The experiments all indicate that the expected control objectives can be achieved and they have the strong stability and anti-interference.

\section{ACKNOWLEDGEMENT}

This research was supported by the Science and Technology Funds from Liaoning Education Department (No. L2015375).

\section{REFERENCES}

[1] Lei Zhang, Xiaomei Cong, Hujian Pan, Zuge Cai, Xiumin Yang, The Control System Modeling and The Mechanical Structure Analysis For EMCVT, TELKOMNIKA, 2013, 11(7): 4159-4167

[2] Toru Fujii, Takemasa Kurokawa and Shigeru Kanehare. A Study of a Metal Pushing V-Belt Type CVT--Part 1: Relation between Transmitted Torque and Pulley Thrust, SAE, Tech. Paper Series 930666(1993)

[3] Naishi Cheng et al. Automotive metal V-belt Type CVT: CVT principles and design, Beijing: Machinery Industry Press, 2007 